\title{
Trombocitopenia grave e infección por virus de inmunodeficiencia humana. Comunicación de dos casos y revisión de la literatura
}

\author{
Rolando Pizarro P., Lucia Aguad R. y Juan L. Castro G.
}

\section{Severe thrombocytopenia and human immunodeficiency virus infection. Report of two cases and review}

Thrombocytopenia is a relatively frequent complication in patients infected by human immunodeficiency virus (HIV). Most frequent mechanisms of thrombopenia are destruction of half-filled platelets by immunocomplex and defects in production. We present two cases of severe thrombocytopenia associated to HIV infection. Case 1: A male patient, 45 years old with fever and diarrhea that lasted for 1 month that presented with thrombopenia of 3.000 platelets $/ \mathrm{mm}^{3}$. After beginning zidovudine and lamivudine therapy, he normalized the platelet count in 5 days. Case 2: A male patient of 30 years old, who suffered during one day migraine, nausea, vomits and then seizures. A criptococccal meningitis was confirmed. Concomitantly he had a platelet count of $59.000 / \mathrm{mm}^{3}$. He started antiretroviral therapy with zidovudina and lamivudina, then was changed to didanosine plus stavudine plus nevirapine. After 6 months of severe thrombocytopenia, platelets count was restored to normal values. A literature review is presented.

Key words: Thrombocytopenia, platelets, immune-complex.

Palabras claves: Trombocitopenia, plaquetas, inmuno-complejo.

\section{Introducción}

S e ha descrito la trombocitopenia (recuento de plaquetas inferior a $150.000 / \mathrm{mm}^{3}$ ) como una complicación relativamente frecuente en el síndrome de inmunodeficiencia adquirida (SIDA) en sus diferentes etapas ${ }^{1,2}$. Las causas más importantes de trombocitopenia en esta situación clínica son ${ }^{1,3-9}$ : de origen periférico, como sucede en la destrucción mediada por inmuno-complejos en un cuadro semejante e indistinguible al púrpura trombocitopénico idiopático (PTI), el hiperesplenismo, las microangiopatias trombóticas o la coagulación intravascular diseminada; y de origen central, como sucede en los defectos de producción causados por la acción directa del virus de la inmunodeficiencia adquirida (VIH) sobre los megacariocitos, la infiltración neoplásica o infecciosa (Pneumocystis jiroveci, Mycobacterium tuberculosis, Mycobacterium avium complex, citomegalovirus) de la médula ósea, la desnutrición y la acción de fármacos. Al asociarse la acción directa del VIH sobre la médula ósea y la generación de auto-anticuerpos, se conforma la llamada trombocitopenia primaria por el virus de la inmunodeficiencia adquirida ${ }^{1,3}$.
Se discute acaso existe una relación directa entre el grado de inmunosupresión, la cuantía de la trombocitopenia y una baja sobrevida, con experiencias contradictorias $^{10}$.

El estudio regular de la trombocitopenia incluye hemograma completo, con recuento y morfología plaquetaria. La biopsia medular se justifica cuando la etiología es oscura. Otros estudios de utilidad son la complementemia, ELISA anti-VHC, la pesquisa de los agentes infecciosos antes mencionados y la determinación de anticuerpos anti-plaquetarios.

Ante la ausencia de publicaciones nacionales relativas a este tema, nos pareció importante comunicar nuestra experiencia en dos casos de asociación entre trombocitopenia e infección por VIH ocurridos el año 2001 y efectuar una revisión de la literatura médica relativa a su tratamiento.

Caso 1. Varón de 45 años, portador de infección por VIH, reconocido desde 1996, en profilaxis con isoniacida y cotrimoxazol. Ingresó a nuestro servicio tras un mes de fiebre intermitente, diarrea, compromiso general y una baja de peso no cuantificada. Al examen físico eran destacables su buen estado de conciencia, tem-
Hospital de Enfermedades Infecciosas Dr. Lucio Córdova L. Santiago, Chile. (RPP, LAR). Hospital Barros Luco Trudeau. Santiago, Chile. Servicio de Hematología (JLCG)

Recibido: 24 junio 2005 Aceptado: 3 agosto 2006

Correspondencia a: Rolando Pizarro Prado enna.zunino@redsalud.gov.cl 
peratura de $37,6^{\circ} \mathrm{C}$, presión arterial $100 / 60 \mathrm{mmHg}$, pulso: 96/min, peso $71 \mathrm{~kg}$ y leve palidez. El examen segmentario sólo evidenció una candidiasis oral, el resto era normal. Exploración de laboratorio: hematocrito $34 \%$, hemoglobina $11 \mathrm{gr} / 1$, leucocitos $2.900 / \mathrm{mm}^{3}$, plaquetas $3.000 / \mathrm{mm}^{3}$, cultivos de LCR, orina y sangre negativos, ADA en sangre 40,7 U/1, LDH en sangre $231 \mathrm{U} / 1, \mathrm{VDRL}$ reactivo débil en sangre y no reactivo en LCR, carga viral de 48.000 copias ARN/ml y linfocitos CD4 de 178 céls $/ \mathrm{mm}^{3}$.

Inició profilaxis con cotrimoxazol y nistatina oral en colutorios por la candidiasis. Se le efectuaron dos transfusiones de plaquetas de $6 \mathrm{U} \mathrm{c} / \mathrm{u}$, con lo cual se elevó el recuento a 10.000 plaquetas $/ \mathrm{mm}^{3}$. Paralelamente, se inició terapia anti-retroviral (TARV) con zidovudina más lamivudina, observándose un incremento a 320.000 plaquetas $/ \mathrm{mm}^{3}$ en una semana. El paciente fue dado de alta, sin que volviera a presente trombocitopenia en controles posteriores. La TARV se mantuvo por dos años más, pero con una evolución inmunológica y virológica desfavorable que determinaron su cambio por didanosina + stavudina + indinavir, esquema que mantiene hasta el momento de este reporte.

Caso 2. Varón de 30 años, portador de infección por VIH, detectada en 1994. Antecedentes de carga viral 95.000 copias ARN/ml y linfocitos CD4 54 céls $/ \mathrm{mm}^{3}$. Inició zidovudina más lamivudina un mes antes de ser hospitalizado. Un día antes de ser internado presentó cefalea, náuseas y vómitos, agregándose un episodio de convulsión tónico clónica. El estudio de LCR reveló la presencia de Cryptococcus neoformans, iniciándose terapia con anfotericina-B deoxicolato, la que posteriormente fue cambiada por fluconazol. El hemograma de ingreso mostraba bicitopenia: hematocrito $31 \%$, hemoglobina $10,5 \mathrm{gr} / 1$, leucocitos $8.200 / \mathrm{mm}^{3}$, plaquetas $59.000 / \mathrm{mm}^{3}$. El mielograma fue informado como: "densidad celular grado II. Médula hipoplásica en todas sus series, sin elementos atípicos. Conclusión: hipoplasia medular, probablemente secundaria a virus de la inmunodeficiencia humana". No se efectuó estudio microbiológico de la médula ósea. Por la buena evolución clínica fue dado de alta con 27 días de hospitalización. Controles posteriores mostraron la persistencia de la bicitopenia en rangos moderados. Por la pobre respuesta virológica e inmunológica, un año y medio después se cambió de TARV a didanosina más stavudina más nevirapina. Pero tres semanas más adelante, el paciente debió ser rehospitalizado por tener un recuento de 10.000 plaquetas $/ \mathrm{mm}^{3}$, bajando hasta $800 /$ $\mathrm{mm}^{3}$; por ello fue transfundido con $6 \mathrm{U}$ de plaquetas una vez. Se suspendió la TARV en curso y se inició prednisona $1 \mathrm{mg} / \mathrm{kg}$ más zidovudina. Las plaquetas experimentaron un alza progresiva siendo dado de alta en breve. Tres meses más tarde se suspendió la zidovudina y reinició la TARV triasociada previa. Se redujo lentamente la prednisona siendo retirada a los 7 meses de su inicio. Los últimos controles revelaron normalización de todos los parámetros hematológicos, con recuentos plaquetarios sobre $160.000 / \mathrm{mm}^{3}$.

\section{Discusión}

Los dos casos relatados corresponden a una etapa en el desarrollo de la TARV en nuestro país, en que el acceso a ella era muy limitado por razones económicas y se disponía de una escasa variedad de antiretrovirales, y con una menor eficacia que los esquemas de alta efectividad existentes en la actualidad.

Ambos pacientes tenían una infección por VIH de larga evolución antes de debutar con un cuadro de trombocitopenia severa. Aunque esta condición se aprecia en todas las etapas de la enfermedad por VIH, a medida que progresa su incidencia se eleva de $3 \%$ hasta más del $40 \%$ en etapa de SIDA avanzado $2,3,8,9$.

En el caso 1, ante la premura de iniciar la TARV no se realizó un estudio acabado que permitiera determinar la etiología de su trombocitopenia; pese a ello, la respuesta clínica al esquema indicado fue tan dramática que se puede deducir como causa de la trombocitopenia, un mecanismo periférico rápidamente controlado. Con el caso 2 se hizo un estudio de la médula ósea que demostró la presencia de atrofia megacariocítica atribuida al VIH, por no encontrarse otros factores etiológicos que pudieran explicarla.

Tanto el inicio precoz de TARV, como la indicación de transfusiones de plaquetas, están supeditadas al recuento plaquetario: sobre $50.000 / \mathrm{mm}^{3}$ no debería adoptarse conducta alguna; entre 49.000 y $20.000 / \mathrm{mm}^{3}$ se recomienda iniciar tratamiento en caso de sangramiento; bajo $20.000 / \mathrm{mm}^{3}$ se recomienda iniciar TARV altamente efectiva ${ }^{11}$.

La evolución de ambos casos marchó por caminos diferentes. En el primero se instauró la TARV disponible en ese momento histórico con zidovudina más lamivudina, observándose una reacción casi inmediata. Los estudios han determinado que zidovudina por sí sola puede incrementar los recuentos plaquetarios en la primera semana de administración y normalizarlos en dos a cuatro semanas ${ }^{12-14}$. Su mecanismo de acción sería un incremento de la eritopoyetina secundario a la anemia provocada por este medicamento, lo que estimularía la respuesta megacariocítica y por consiguiente la trombopoyesis, así como por su efecto antiretroviral $^{10,15,16}$. De todas formas, el uso de una TARV combinada o altamente efectiva, es considerada como la primera medida a tomar, en caso de no estar ya en 
curso, para tratar la trombocitopenia grave causada por el $\mathrm{VIH}^{17,18}$.

El segundo caso tuvo una biopsia medular concordante con infiltración medular por VIH, lo que explica su tórpida evolución, con mantención de la bicitopenia en general, y la trombocitopenia en particular, por más de un año y medio. Cuando se decidió cambiar el esquema anti-retroviral, suspendiéndose la zidovudina, se observó una depresión extrema de los trombocitos que bajaron hasta $800 / \mathrm{mm}^{3}$. Debido a esto, se volvió a administrar zidovudina, cuyos efectos ya comentamos, pero esta vez asociada a prednisona en la dosis estándar recomendada de $1 \mathrm{mg} / \mathrm{kg}$ peso ${ }^{17}$. Los corticoesteroides actúan más bien en la periferia, en forma temprana, disminuyendo la actividad del receptor Fc de los macrófagos esplénicos $\mathrm{y}$, tardíamente, disminuyendo también la generación de anticuerpos antiplaquetarios. Por otra parte, es conocida la capacidad de los corticoesteroides como estabilizador de membranas y en estas circunstancias, ayudan a reinstaurar la integridad del endotelio vascular, explicando la disminución de las hemorragias, incluso antes de la recuperación de los recuentos plaquetarios ${ }^{1}$. Aún así, los corticoesteroides son recomendados por tiempos cortos y como fármacos de segunda línea por sus múltiples efectos adversos asociados, entre otros aumento de peso, miopatías, neuropatías, acné, alteraciones mentales y, en especial, la reactivación de infecciones como tuberculosis, sarcoma de Kaposi, candidiasis, herpes simplex, neumonías y hepatitis $\mathrm{B}$, entre otras ${ }^{19,20}$. No obstante este riesgo, nuestro paciente recibió prednisona durante siete meses y no presentó complicación secundaria alguna.

El uso de transfusiones plaquetarias es una medida de urgencia, recomendada con recuentos inferiores a $10.000 / \mathrm{mm}^{3}$ en ausencia de sangramiento, como sucedió en estos dos casos y con recuentos bajo 20.000/ $\mathrm{mm}^{3}$ si el paciente está sangrando ${ }^{1,11}$.

Otras medidas, que no se utilizaron en estos casos pero que es necesario considerar son:

- Uso de inmunoglobulina intravenosa (IGIV). Diversos estudios han demostrado su eficacia en dosis entre 0,5 y $2 \mathrm{~g} / \mathrm{kg}$, administrada en esquemas de 4 y 1 día, respectivamente. Se ha observado la reactivación de la trombocitopenia a las tres semanas de suspender el preparado, ante lo cual se consideran administraciones cada dos semanas ${ }^{21-23}$. La IGIV actuaría inhibiendo los receptores $\mathrm{Fc}$ con lo cual disminuye la acción macrofágica sobre los trombocitos y previene la formación de complejos inmunes citopáticos ${ }^{24-26}$. Es una terapia costosa y de disponibilidad limitada.

- La inmunoglobulina anti Rh ha demostrado su utilidad en pacientes Rh positivos no esplenectomi- zados. Al unirse este preparado al antígeno D de los eritrocitos, permite que éstos sean atrapados por el bazo disminuyendo la captura y destrucción de plaquetas $^{27}$. Se usa en dosis de $50 \mathrm{mg} / \mathrm{kg}$ al día durante una semana y luego es repetida cada tres semanas. Puede producir hemólisis, por ello se debe vigilar de cerca su administración, en especial si coexiste con anemia ${ }^{27,28}$. Tiene la ventaja de ser más económica que la IGIV.

- Esplenectomía, indicada en una trombocitopenia persistente, con fracaso de las terapias antes mencionadas. La respuesta a largo plazo ha sido satisfactoria en distintos estudios ${ }^{29-31}$. Su mecanismo de acción es la eliminación de un órgano clave en la destrucción de las plaquetas unidas a inmuno-complejos y generador de anticuerpos anti-plaque$\operatorname{tarios}^{30,32}$. La esplenectomía ocasiona, como efecto residual, un estado de inmunosupresión (desaparición de la fagocitosis intraesplénica) que favorece la aparición de sepsis fulminantes causadas por Streptococcus pneumoniae, Neisseria meningitidis y Haemophilus influenzae tipo $\mathrm{b}^{32,33}$. Está recomendado efectuar la vacunaciones correspondientes antes del procedimiento quirúrgico.

- Interferón alfa, se usan 3.000.000 U/3 veces a la semana, con rápida recuperación en el recuento plaquetario; a las pocas semanas de su suspensión nuevamente las plaquetas caen a sus valores basales $^{34}$. Se indica en caso de fracasar la TARV, las inmunoglobulinas o la esplenectomía.

- Algunos estudios han demostrado la utilidad de la trombopoyetina en estimular los recuentos de plaquetas pero tiene la desventaja de observarse una rápida caída al suspender su $\mathrm{uso}^{35}$.

- La infusión de factor humano de crecimiento megacariocítico pegilado recombinante ha demostrado ser útil para restaurar el recuento plaquetario, a la vez que disminuye la susceptibilidad de tales plaquetas a los anticuerpos anti-plaquetarios de acción periférica ${ }^{36}$. Se necesitan estudios con seguimiento a largo plazo para tener una mejor valoración de este producto.

Ante la presencia de una trombocitopenia en un paciente con infección por VIH, debe ponderarse la intensidad de ésta, así como la etapa de progresión del SIDA para tomar decisiones en lo relativo al estudio a realizar, como en lo referente a la estrategia terapéutica a seguir. Los dos casos comunicados presentaron trombocitopenia grave asociada al VIH; aunque la evolución, el estudio efectuado, la terapia y la respuesta a ésta fueron distintos; ambos nos han permitido revisar los mecanismos patogénicos descritos y las alternativas de manejo recomendadas en estas circunstancias. 


\section{Resumen}

La trombocitopenia es una complicación relativamente frecuente en los pacientes infectados por el virus de la inmunodeficiencia humana. Las mecanismos más frecuentes de génesis son la destrucción de trombocitos mediada por inmunocomplejos y los defectos en su producción. Presentamos dos casos de trombocitopenia grave asociados a infección por VIH. Caso 1. Varón de 45 años que consultó por cuadro febril y diarreico de un mes de evolución y trombocitopenia: 3.000 plaquetas $/ \mathrm{mm}^{3}$. Normalizó su recuento plaquetario al $5^{\circ}$ día de iniciar zidovudina + lamivudina. Caso 2. Varón de 30 años, consultó por cefalea, náuseas, vómitos y convulsiones. Se confirmó la presencia de meningitis por Cryptococcus sp. El recuento plaquetario era de $59.000 / \mathrm{mm}^{3}$. Recibió zidovudina + lamivudina, luego didanosina + stavudina + nevirapina; tras 6 meses de trombocitopenia grave, normalizó el recuento plaquetario. Se presenta revisión de la correspondiente literatura científica.

\section{Referencias}

1.- Zunino J, Torales M. Trombocitopenia en infectados por el virus de la inmunodeficiencia humana. http://www. aidsmap.com/en/main/sitemap.asp?404=true (accedido en abril de 2005)

2.- Morris L, Distenfeld A, Amorosi E, Karpatkin S. Autoimmune thrombocytopenic purpura in homosexual men. Ann Intern Med 1982; 96: 714-7.

3.- Conte G, Araos D. Manifestaciones hematológicas. En Sepúlveda C, Afani A, editores. SIDA. $3^{\text {a }}$ edición, 2002. Santiago de Chile. Mediterráneo. pp: 282-310.

4.- Walsh C M, Nardi M A, Karpatkin S. On the mechanism of thrombocytopenic purpura in sexually active homosexual men. N Engl J Med 1984; 311: 635-9.

5.- Walsh C, Krigel R, Lennette E, Karpatkin S. Thrombocytopenia in homosexual patients. Ann Intern Med 1985; 103: 542-5.

6.- Cole J L, Marzec U M, Gunthel C J, Karpatkin S, Worford L, Sundell I B, et al. Ineffective platelet production in thrombocytopenic human immunodeficiency virus-infected patients. Blood 1998; 91: 3239-46.

7.- Louache F, Bettaieb A, Henri A, Oksenhendler E, Farcet P, Bierling D, et al. Infection of megakaryocytes by human immunodeficiency virus in seropositive patients with immune thrombocytopenia. Blood 1991; 78: 1697-705.

8.- Moses A, Nelson J, Bagby G C Jr. The influence of human immunodeficiency virus-1 on hematopoiesis. Blood 1998; 91: 1479-95.

9.- Domínguez Castellano A, Vásquez Rodríguez J J. Trombocitopenia asociada a la infección por el virus de la inmunodeficiencia humana. Med Clin (Barc) 1992; 98: 671-5.

10.- Glatt A, Anand A. Thrombocytopenia in patients infected with HIV: Treatment update. Clin Infect Dis 1995; 21: 415-23.
11.- Coyle T. Hematologic complications of human immunodeficiency virus infection and the acquired immunodeficiency syndrome. Med Clin North Am 1997; 81: 449-70.

12.- Hymes K B, Greene J B, Karpatkin S. The effect of azidothymidine on HIV-related thrombocytopenia. N Engl J Med 1988; 318: 516-7.

13.- The Swiss Group for Clinical Studies on the Acquired Immunodeficiency Syndrome (AIDS). Zidovudine for the treatment of thrombocytopenia associated with human immunodeficiency virus (HIV) infection. A prospective study. Ann Intern Med 1988; 109: 718-21.

14.- Oksenhendler E, Bierling P, Ferchal F, Clauvel J P, Seligmann M. Zidovudine for thrombocytopenic purpura related to human immunodeficiency virus infection. Ann Intern Med 1989; 110: 365-8.

15.- Fischl M A, Richman D D, Grieco M H, Gottlieb M S, Volberding P A, Laskin O L. The efficacy of azidothymidine (AZT) in the treatment of patients with AIDS and AIDS-related complex: a double-blind, placebo-controlled trial. N Engl J Med 1987; 317: 185-91.

16.- Burstein S A, Ishibashi T. Erythropoietin and megakaryocytopoiesis. Blood Cells 1989; 15: 193-201

17.- Caso J A, Mingo C S, Tena J G. Effect of highly active antiretroviral therapy on thrombocytopenia in patients with HIV infection. N Engl J Med 1999; 341: 1239-40.

18.- Maness L, Blair D, Newman N, Coyle T. Elevation of platelet counts associated with indinavir treatment in human immunodeficiency virus-infected patients. Clin Infect Dis 1998; 26: 207-8.

19.- Abrams D I, Kirpov D D, Geodert J, Sarngadharan M G, Gallo R C, Volberding P A. Antibodies to human T-lymphotropic virus type III and development of the acquired immunodeficiency syndrome in homosexual men presenting with immune thrombocytopenia. Ann Intern Med 1986; 104: $47-50$.

20.- Shafer R W, Offit K, Macris N T, Horbar G M, Ancona L, Hoffman I R. Possible risk of steroid administration in patients at risk for AIDS. Lancet 1985; 1 (8434): 934-5.

21.- Pollak A N, Janinis J, Green D. Successful intravenous immune globulin therapy for human immunodeficiency virus-associated thrombocytopenia. Arch Intern Med 1988; 148: 695-7.

22.- Rarick M U, Montgomery T, Groshen S, Sullivan-Halley J, Jamin D, Mazumder A, et al. Intravenous immunoglobulin in the treatment of human immunodeficiency virus-related thrombocytopenia. Am J Hematol 1991; 38: 261-6.

23.- Bussel J B, Haimi J S. Isolated thrombocytopenia in patients infected with HIV: treatment with intravenous gammaglobulin. Am J Hematol 1988; 28: 79-84.

24.- Bussel J B, Kimberly R P, Inman R D, Schulman I, Cunnigham-Rundles, Cheung N, et al. Intravenous gammaglobulin treatment of chronic idiopathic thrombocytopenic purpura. Blood 1983; 62: 480-6.

25.- Newland A C, Trleaven J G, Minchinton R M, Waters A H. High-dose intravenous IgG in adults with autoimmune thrombocytopenia. Lancet 1983; 1: 84-7.

26.- Fehr J, Hofman V, Kappeler U. Transient reversal of thrombocytopenia in idiopathic thrombocytopenia purpura by high-dose intravenous gamma globulin. N Engl J Med 1982; 306: 1254-8.

27.- Bussel J B, Graziano J N, Kimberly R P, Pahwa S, Aledort L M. Intravenous anti-D treatment of immune thrombocytopenic purpura: analysis of efficacy, toxicity and mechanism of effect. Blood 1991; 77: 1884-93.

28.- Oksenhendler E, Bierling P, Brossard Y, Schemmetzler C, Girard P M, Seligmann M, 
et al. Anti-Rh immunoglobulin therapy for human immunodeficiency virus-related immune thrombocytopenic purpura. Blood 1988; 71:1499.

29.- Schneider P A, Abrams D I, Rayner A A, Hohn D C. Immunodeficiency-associated thrombocytopenic purpura (IDTP):

Response to splenectomy. Arch Surg 1987; 122: $1175-78$.

30.- Ravikumar T S, Allen J D, Bothe A Jr, Steele G Jr. Splenectomy. The treatment of choice for human immunodeficiency virusrelated immune thrmbocytopenia? Arch Surg. 1989; 124: 625-8.

31.- Oksenhendler E, Bierling P, Chevret S,
Delfraissy J, Laurian Y, Clauvel J P, et al. Splenectomy is safe and effective in human immunodeficiency virus-related immune thrombocytopenia. Blood 1993; 82: 3504-5.

32.- Kim H C, Raska K, Trooskin S, Saidi P. Immune thrombocytopenia in hemophiliacs infected with human immunodeficiency virus and their response to splenectomy. Arch Intern Med 1989; 149: 1685-8.

33.- Dickerman J D. Splenectomy and sepsis: a warning. Pediatrics 1979; 63: 939-41.

34.- Marroni M, Gresele P, Landonio G, Lizzarin A, Coen M, Vezza R, et al. Interferon a is effective in the treatment of HIV-1 related, severe, zidovudine-resistant thrombocytopenia. A prospective placebocontrolled double-blind trial. Ann Intern Med 1994; 121: 423-9.

35.- Harker L A Carter R A, Marzec U M, Novembre F, Sundell B, Waller E, et al. Correction of thrombocytopenia and ineffective platelet production in patients infected with human immunodeficiency virus (HIV) by PEG-rHuMG. Blood 1998; 92: 707a (abstr).

36.- Sundell I B, Koka PS. Thrombocytopenia in HIV infection: impairment of platelet formation and loss correlates with increased c-Mpl and thrombopoietin expression. Curr HIV Res 2006; 4: 107-16. 